\title{
Terminal Electron-Proton Transfer Dynamics in the Quinone Reduction of Respiratory Complex I
}

\author{
Ana P. Gamiz-Hernandez, ${ }^{* \dagger}$ Alexander Jussupow, ${ }^{\dagger}$ Mikael P. Johansson, $^{\dagger}{ }^{\ddagger}$ a and Ville R. I. Kaila ${ }^{*}{ }^{\dagger}$ \\ ${ }^{\dagger}$ Department Chemie, Technische Universität München (TUM), Lichtenbergstraße 4, Garching D-85747, Germany \\ ${ }^{\ddagger}$ Department of Chemistry, University of Helsinki, P.O. Box 55, Helsinki FI-00014, Finland
}

\section{Supporting Information}

ABSTRACT: Complex I functions as a redox-driven proton pump in aerobic respiratory chains. By reducing quinone (Q), complex I employs the free energy released in the process to thermodynamically drive proton pumping across its membrane domain. The initial $Q$ reduction step plays a central role in activating the proton pumping machinery. In order to probe the energetics, dynamics, and molecular mechanism for the proton-coupled electron transfer process linked to the $\mathrm{Q}$ reduction, we employ here multiscale quantum and classical molecular simulations. We identify that both ubiquinone (UQ) and menaquinone (MQ) can form stacking and hydrogen-bonded interactions with the conserved Qbinding-site residue His-38 and that conformational changes between these binding modes modulate the $\mathrm{Q}$ redox potentials and the rate of electron transfer (eT) from the terminal N2 iron-sulfur center. We

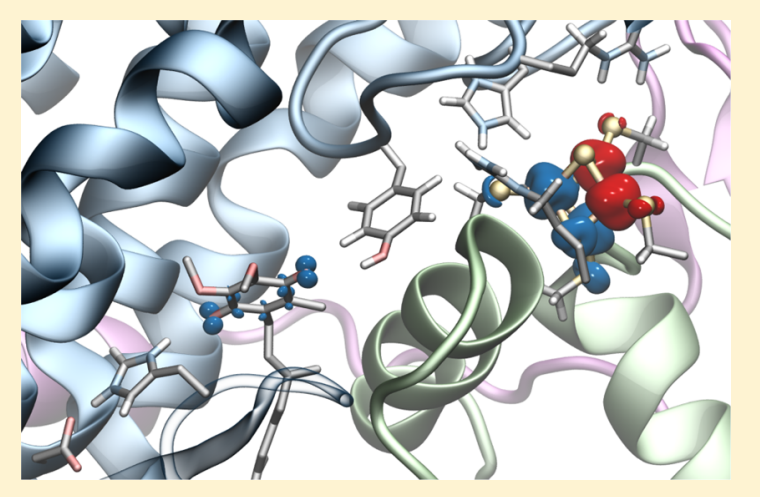
further observe that, while the transient formation of semiquinone is not proton-coupled, the second eT process couples to a semiconcerted proton uptake from conserved tyrosine (Tyr-87) and histidine (His-38) residues within the active site. Our calculations indicate that both UQ and MQ have low redox potentials around -260 and $-230 \mathrm{mV}$, respectively, in the Q-binding site, respectively, suggesting that release of the $Q$ toward the membrane is coupled to an energy transduction step that could thermodynamically drive proton pumping in complex I.

\section{INTRODUCTION}

Respiratory complex I (NADH:ubiquinone oxidoreductase) is a redox-driven proton pump that reduces quinone $(\mathrm{Q})$ by electron transfer (eT) from nicotinamide adenine dinucleotide $(\mathrm{NADH})$ and couples the energy released in the process to transfer of protons ( $\mathrm{pT}$ ) across the mitochondrial or bacterial membrane, ${ }^{1-7}$ (Figure 1). The eight to nine iron-sulfur centers (ISCs) of the hydrophilic domain of complex I are responsible for catalyzing the $\mathrm{eT}$ reaction, ${ }^{8}$ while the proton pumping entirely takes place in the membrane domain of the enzyme. ${ }^{1,3-6}$ Remarkably, the most distant subunits responsible for the pT are separated by up to ca. $200 \AA$ from the eT domain. ${ }^{9-14}$ This long-range coupling is believed to take place by combined conformational and electrostatic transitions, in which the terminal eT step between the N2 ISC and Q is likely to trigger the proton-pumping activity, ${ }^{1,3-6,8-18}$ but the exact molecular mechanism remains elusive.

Although the exact binding mode of $Q$ has not been experimentally resolved in complex I, structural and biochemical studies ${ }^{2,9-14}$ show that the Q-binding site has a unique location in complex I. ${ }^{19}$ In contrast to many membrane proteins, in which the $Q$ site is embedded within the membrane domain, the $Q$ site in complex I is located ca. $20 \AA$ above the membrane plane and ca. $8-11 \AA$ from the terminal N2 ISC ${ }^{20}$ (see Figure S1 for nomenclature of ISCs in complex I), with Tyr- $87^{21}$ and His-38 interacting with the Q headgroup (Figure
1). ${ }^{22}$ Although proton-coupled electron transfer (PCET) reactions of Qs in different solvents and oxidoreductases have been intensively studied over the years, ${ }^{23-27}$ a detailed understanding of the Q-reduction-linked PCET processes in complex I is still unclear. Recent electrochemical studies ${ }^{28}$ indicate that the redox potential of ubiquinone (UQ) for the $\mathrm{Q} / \mathrm{QH}_{2}$ redox couple is unusually low, in the $-300 \mathrm{mV}$ range, which is considerably downshifted from the $E_{\mathrm{m}}$ of $\mathrm{Q}$ in membranes of ca. $+90 \mathrm{mV}$; electron paramagnetic resonance studies $^{8,29}$ show that the $E_{\mathrm{m}}$ of the terminal N2 ISC is ca. -150 $\mathrm{mV}$ in Escherichia coli (all $E_{\mathrm{m}}$ values are relative to NHE if not otherwise stated). Moreover, experiments suggest $^{28}$ that semiquinone (SQ) does not accumulate during turnover (but cf. ref 30), further supporting that the SQ species is thermodynamically unstable and transient. Interestingly, recent experiments $^{19}$ also indicate that the $\mathrm{eT}$ rate in complex I may be modulated by the redox state of the terminal N2 cluster, which in turn may be important for understanding the eT dynamics along the ISC chain. Importantly, elucidating the thermodynamics of the terminal eT step that is linked to the $\mathrm{Q}$ reduction in complex $\mathrm{I}$ is important for understanding how the redox energy is employed for proton pumping.

Received: August 16, 2017

Published: October 10, 2017 


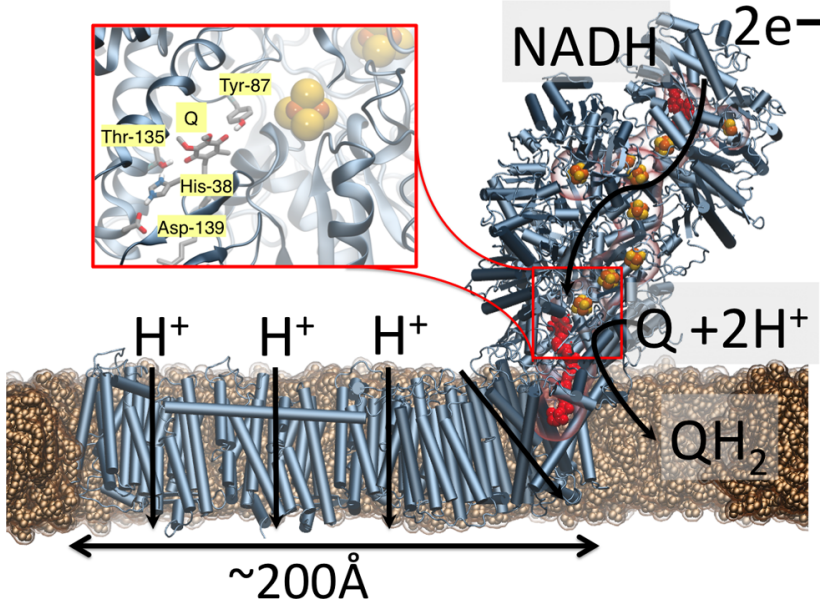

Figure 1. Complex I, the initial electron acceptor in aerobic respiratory chains. Electron transfer from $\mathrm{NADH}$ to $\mathrm{Q}$ via eight to nine ISCs leads to pumping of protons in the membrane domain of complex I. (inset) $\mathrm{Q}$ and N2 sites are located about $20 \AA$ above the membrane plane; Q has been modeled in computationally (see the Computational Methods section).

In order to address the mechanism of the Q reduction by the terminal $\mathrm{N} 2$ cluster and the $E_{\mathrm{m}}$ of $\mathrm{Q}$ in complex $\mathrm{I}$, we performed here first-principles quantum chemical calculations (QM), hybrid quantum mechanics/classical mechanics (QM/ $\mathrm{MM}$ ) simulations, classical molecular dynamics (MD) simulations, as well as continuum Poisson-Boltzmann (PB) electrostatics calculations on molecular models that are based on the experimentally resolved structure of complex I from Thermus thermophilus, ${ }^{11}$ for which no Q-bound structure has yet been released. Q-binding models were constructed for both UQ and menaquinone (MQ), which complex I can employ as a substrate, for example, under anaerobic conditions. ${ }^{31}$ In order to control the localization of the electron between $\mathrm{N} 2$ and $\mathrm{Q}$ we develop here a computational approach, where we initiate the simulations from reduced or oxidized QM subsystems that are created by merging converged electronic structures from the individual donor, $\mathrm{N} 2$, and acceptor, $\mathrm{Q}$, subsystems together. This allows us to study the energetics and dynamics of the eT process from localized diabatic electronic states of the reduced/ oxidized N2 and Q. Our combined results identify important UQ- and MQ-binding configurations within the Q-binding site of complex I. We also characterize the $E_{\mathrm{m}}$ of UQ/MQ in these states and show how the terminal eT from $\mathrm{N} 2$ is linked to coupled protonation changes of nearby residues.

\section{RESULTS AND DISCUSSION}

2.1. Binding Modes of $Q$ in the Active Site of Complex I. Our hybrid QM/MM simulations, quantum chemical density functional theory (DFT) models, as well as classical MD simulations of UQ and MQ in different oxidation states show that both species can bind in two possible conformations within the binding pocket, shown in Figure 2 for UQ and Figure S3 for MQ. In the hydrogen-bonded binding mode, UQ/MQ forms a hydrogen-bonded interaction with both His-38 and Tyr-87, while in the stacking binding mode, UQ/MQ interacts dispersively with His-38, while retaining the hydrogen bond with Tyr-87. Comparison of computed IR spectra of these conformations predict a ca. $200 \mathrm{~cm}^{-1}$ shift on the His-38 $\mathrm{N} \varepsilon-\mathrm{H}$ bond, which could be employed to spectroscopically identify the two binding poses (Figure S7) by using, for example,
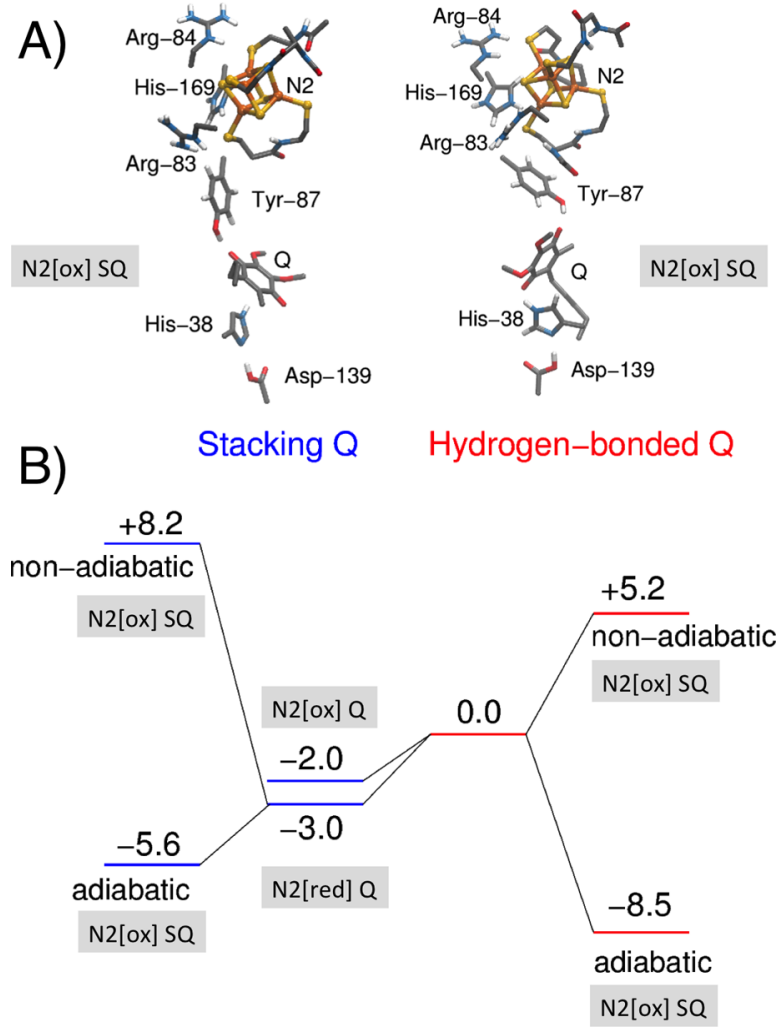

Figure 2. (A) Structure and (B) B3LYP-D3/def2-TZVPP $/ \varepsilon=4$ energetics (in $\mathrm{kcal} \mathrm{mol}^{-1}$ ) of hydrogen-bonded (right) and stacked (left) binding modes of UQ in complex I. Q and SQ refer to oxidized quinone and semiquinone species, while $\mathrm{N} 2[\mathrm{ox}]$ and $\mathrm{N} 2$ [red] refer to oxidized $(2 \mathrm{Fe}[\mathrm{II}] 2 \mathrm{Fe}[\mathrm{III}])$ and reduced $(3 \mathrm{Fe}[\mathrm{II}] 1 \mathrm{Fe}[\mathrm{III}])$ states of $\mathrm{N} 2$, respectively. Nonadiabatic and adiabatic eT energetics are calculated by relaxing the initial electron donor state $(\mathrm{N} 2 / \mathrm{Q})$ or by relaxing both the initial $(\mathrm{N} 2[\mathrm{red}] / \mathrm{Q})$ and final $(\mathrm{N} 2[\mathrm{ox}] / \mathrm{SQ})$ states, respectively.

Fourier-transform IR methodology as applied for Photosystem II. ${ }^{32}$ While UQ swaps between these two binding modes on nanosecond time scales in the oxidized state (Figure S2), MQ forms a stronger stacking interaction with His-38 in the oxidized state and favors more strongly the hydrogen-bonded interaction in the SQ state. This one-electron reduced UQ/MQ species forms an anionic semiquinone $\left(\mathrm{Q}^{\bullet /-}\right)$, without proton abstraction of the surrounding residues, consistent with previous results. ${ }^{17}$ Our QM calculations further indicate that the stacked $\mathrm{Q}$ conformation is favored by ca. $2 \mathrm{kcal} \mathrm{mol}^{-1}$ over the hydrogen-bonded binding mode and is weakly affected by the reduction of N2 (Figures 2 and S18). On the basis of the computed energetics, we expect that the hydrogen-bonded mode would have a low occupation, while structural studies might identify only the stacked conformation. In contrast to the oxidized UQ we find that the SQ favors the hydrogen-bonded binding mode by ca. $2 \mathrm{kcal} \mathrm{mol}^{-1}$ (Table S1), whereas the menasemiquinone (i.e., $\mathrm{MQ}^{\bullet /-}$ ) favors the hydrogen-bonded binding mode by ca. $5 \mathrm{kcal} \mathrm{mol}^{-1}$ due to the stronger dispersive $\pi-\pi$ interaction between His-38 and the MQ rings in the oxidized state (Table S2). Our QM/MM calculations support these results (Figure 3), and a similar behavior is also found in our classical MD simulations (Figure S2). Structural comparison of the two binding modes for the DFT cluster models (Figure S4) shows that there is little variation between the $Q$ and the $\mathrm{N} 2$ distances, but our MD simulations indicate that, in 

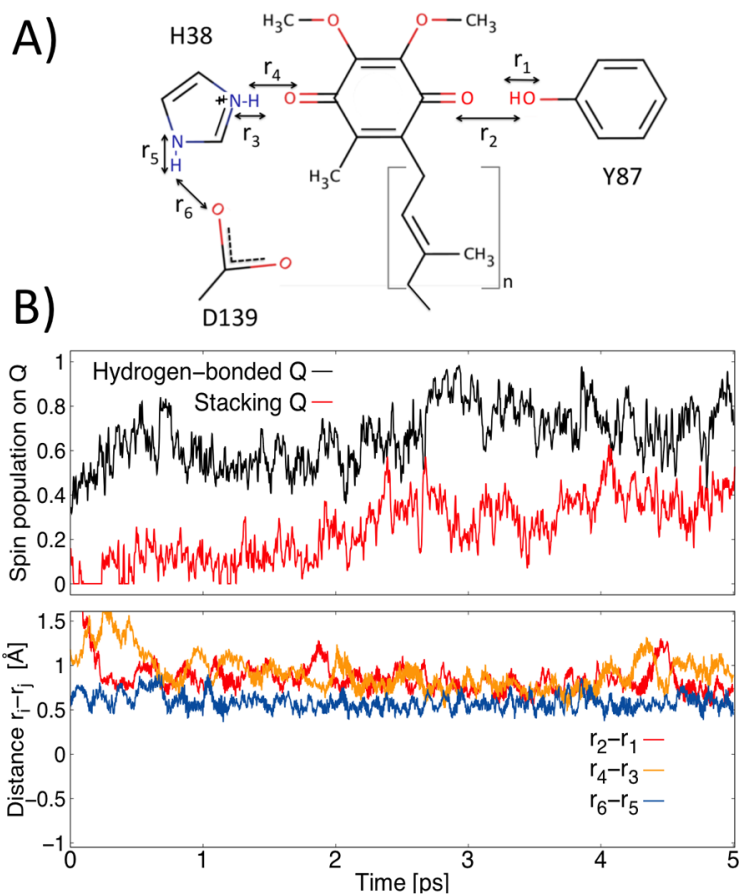

Figure 3. (A) pT distances between $\mathrm{UQ}$ and the proton donors, Y87 $\left(r_{2}-r_{1}\right)$ and H38 $\left(r_{4}-r_{3}\right)$, as well as between H38 and D139 $\left(r_{6}-r_{5}\right)$. (B) $\mathrm{QM} / \mathrm{MM}$ dynamics of eT between $\mathrm{N} 2$ and UQ in the stacked (in red) and hydrogen-bonded conformations (in black). The hydrogenbonded conformation leads to formation of $S Q$ indicated by the higher spin population relative to the stacked conformation. The fractional spin occupation on the UQ in the stacked conformation might result from DFT charge transfer problems ${ }^{38}$ (see Figure S12). The early steps of eT indicate that, within the first $0.5 \mathrm{ps}$, the hydrogen bonds with $\mathrm{Y} 87$ and $\mathrm{H} 38$ become stronger but no pT is observed.

the stacked conformation, the $\mathrm{N} 2-\mathrm{Q}$ distance is ca. $0.5 \AA$ shorter than in the hydrogen-bonded conformation (Figures S5 and S6), which could affect the rate of eT from N2 (see below).

2.2. Energetics of eT between $\mathbf{N} 2$ and $\mathrm{Q}$. By combining DFT (B3LYP-D3) molecular orbitals obtained from the UQ (or MQ) and N2 broken-symmetry spin-flip subsystems, we converged the electronic structure into localized diabatic electronic states of reduced/oxidized N2 and Q/SQ (MQ/ MSQ). We find that the UQ reductions become thermodynamically favored by ca. $-9 \mathrm{kcal} \mathrm{mol}^{-1}$ in the hydrogen-bonded conformation as compared to ca. $-4 \mathrm{kcal} \mathrm{mol}^{-1}$ in the stacked conformation. This indicates that the first eT step may couple to a conformational change of the $\mathrm{Q}$ from the stacked to the hydrogen-bonded binding mode. The conformational change is also reflected in the rather large nonadiabatic eT energy gap of ca. $11 \mathrm{kcal} \mathrm{mol}^{-1}$, disfavoring formation of $\mathrm{SQ}$ which is obtained without relaxing the geometry of the electronaccepting $Q$ subsystem. In contrast, the nonadiabatic eT energy is reduced to ca. $5 \mathrm{kcal} \mathrm{mol}^{-1}$ in the hydrogen-bonded binding mode. This suggests that the thermodynamics of the eT between $\mathrm{N} 2$ and $\mathrm{Q}$ is nearly degenerate in the stacked conformation (Table S1). We find that the various spin-state configurations, that is, different broken-symmetry ${ }^{33}$ combinations of the unpaired electrons around the individual high-spin iron centers leading to the same total spin at the ISCs, are energetically quite different. We use the lowest energy configuration, but we note that other choices may tune the relative stability of the reduced $\mathrm{N} 2$ center by up to ca. $6 \mathrm{kcal}$ $\mathrm{mol}^{-1}$. Interestingly, in our DFT models of the other ISCs, we do not observe such a large energy difference between different spin-state configurations, which may relate to the uniquely connected cysteine residue, Cys-45/Cys- $46 .^{2}$ Moreover, we observe that the relative stability of SQ is qualitatively similar using other density functionals (Table S1). For MQ the eT from $\mathrm{N} 2$ to $\mathrm{MQ}$ is exergonic by ca. $2 \mathrm{kcal} \mathrm{mol}^{-1}$ and ca. $9 \mathrm{kcal}$ $\mathrm{mol}^{-1}$ in the stacked and hydrogen-bonded binding modes, respectively, suggesting that $\mathrm{Q}$ and $\mathrm{MQ}$ might be redox tuned in a similar way in the Q-binding pocket (Table S2).

In order to probe the effect of the protein environment and estimate $E_{\mathrm{m}}$ values, we performed classical $\mathrm{PB}$ continuum electrostatics calculations with Monte Carlo (MC) sampling of surrounding residues in different protonation/redox states (Figures $\mathrm{S} 8$ and $\mathrm{S} 9$ ). For $\mathrm{UQ}$, we obtain an average $E_{\mathrm{m}}$ for the $\mathrm{Q} / \mathrm{SQ}$ redox couple of $-260 \mathrm{mV}$ with $\mathrm{N} 2[\mathrm{ox}]$ and $-320 \mathrm{mV}$ with $\mathrm{N} 2$ [red] in the hydrogen-bonded conformation that contrast the average $E_{\mathrm{m}}$ of $-380 \mathrm{mV}$ for the stacked $\mathrm{Q}$ conformation with $\mathrm{N} 2[\mathrm{ox}]$. For the $\mathrm{N} 2$ center, we obtain an $E_{\mathrm{m}}$ of $-300 \mathrm{mV}$, which is considerably upshifted by ca. $200 \mathrm{mV}$ from the other ISC of complex I (Figures S8 and S9). Although, somewhat downshifted from the experimental $E_{\mathrm{m}}$ values of $\mathrm{N} 2$ (Table S1), these values are in qualitative agreement with the difference reported for $E_{\mathrm{m}}$ values between $\mathrm{N} 2$ and other ISCs in complex I. ${ }^{20}$ The computed values are not strongly affected by applying other dielectric constants in the PB framework (Figure S10) or by the number of titrated ISCs included in the calculations (Table S4). Moreover, the values are also in qualitative agreement with electron affinities calculated based on the QM cluster models, where we find that the relative electron affinity of $\mathrm{N} 2$ is ca. $200 \mathrm{mV}$ higher than that for a $\mathrm{QM}$ model of the $\mathrm{N} 4$ center model, giving an electron affinity of $\mathrm{N} 2$ in the $-50 \mathrm{mV}$ range, based on the employed experimental $E_{\mathrm{m}}$ of $\mathrm{N} 4$ at ca. $-250 \mathrm{mV}$ (Table S1). Our calculations suggest that the negative charge of the $\mathrm{N} 2$ center downshifts the electron affinity of $Q$ by ca. $400 \mathrm{mV}$, which would place the $E_{\mathrm{m}}$ of $\mathrm{Q}$ near $-300 \mathrm{mV}$, considering a reference value of $+90 \mathrm{mV}$ in membranes.

Similar to UQ we obtain for MQ in its hydrogen-bonded binding mode an $E_{\mathrm{m}}$ value of $-230 \mathrm{mV}$ (Table S5), supporting that the protein environment tunes the redox potentials of both UQ and MQ in a similar way, although MQ has a ca. $170 \mathrm{mV}$ lower $E_{\mathrm{m}}$ value in membranes relative to UQ. ${ }^{34}$ For the stacked $\mathrm{MQ}$ we obtain a strongly downshifted $E_{\mathrm{m}}$, most likely due to the conformational switching between the two conformations upon reduction of MQ.

The $E_{\mathrm{m}}$ values calculated at the PB level are thus consistent with experiments ${ }^{28}$ and the $\mathrm{QM}$ calculations, suggesting that the $\mathrm{Q} / \mathrm{SQ}$ redox couple is less than $-300 \mathrm{mV}$. ${ }^{28,35}$ Interestingly, the PB calculations suggest that reduction of N2 increases the proton affinity of a nearby His-169, consistent with site-directed mutagenesis studies by Zwicker and coworkers $^{36}$ (Table S3). Our calculations thus show that the binding mode of $\mathrm{Q}$ hydrogen-bonded or stacked, may modulate its redox potential, while the binding energetics itself is linked to the redox state of the N2 center. A similar stackinginduced redox-tuning effect has also been suggested for Photosystem I, where a low-potential $Q$ stacks with a tryptophan residue. ${ }^{37}$

We next probed the energetics for the second eT between $\mathrm{N} 2$ and $\mathrm{SQ}$ initiated by re-reduction of N2. Consistent with previous simulations, ${ }^{17}$ our DFT models suggest that the process is coupled to proton abstraction from surrounding Tyr87 residues, whereas no complete proton abstraction from His- 
38 was observed, leading to the effective formation of $\mathrm{QH}^{-}$. We obtain an overall endergonicity of ca. $20 \mathrm{kcal} \mathrm{mol}^{-1}$ in the DFT models, which might result from the protonated His-169 that disfavors the reduction of N2. However, DFT calculations suggest that deprotonation of His- 169 could stabilize this eT by

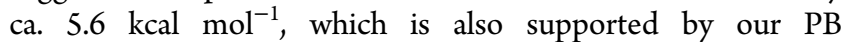
calculations. Taken together, the results suggest that reduction of N2 is linked to deprotonation of His-169 (see below and Table S3), further stabilizing the eT process.

2.3. Simulating the eT Dynamics. In order to address the eT dynamics between N2 and UQ, we performed QM/MM MD simulations, with both UQ and N2 forming the QM region and the remaining system treated classically (Figure S11). Also, for these models, the reduced donor and oxidized acceptor subsystems were generated by merging together their individual molecular orbitals to generate initial $\mathrm{eT}$ states with the electron on N2. The results, shown in Figure 3, indicate that the conformation of $\mathrm{Q}$ indeed modulates the rate of $\mathrm{eT}$, consistent with the results obtained from the QM calculations and the $\mathrm{PB}$ calculations of $E_{\mathrm{m}}$ values. In the hydrogen-bonded conformation, we observe a rapid eT between N2 and UQ (Figure 3), whereas in the stacked conformation the SQ formation is slowed down. On the basis of a Moser-Dutton treatment, ${ }^{39,40}$ by using the calculated $\Delta G$ values $(-40$ and $+80 \mathrm{mV}), \mathrm{N} 2-\mathrm{UQ}$ edge-to-edge distance (10.5 $\AA)$, generic reorganization energy $(\lambda$ $=0.7 \mathrm{eV})$, and protein-packing density $(\rho=0.76)$, we obtain predicted eT rates of ca $1 \times 10^{-7}$ and $6 \times 10^{-7} \mathrm{~s}^{-1}$ for the hydrogen-bonded and stacked conformations, respectively, suggesting that the eT could nevertheless be possible from both binding conformations. However, an accurate estimation of eT requires an explicit calculation of the electronic overlap, which is outside the scope of the present work.

As DFT spin energetics are known to be sensitive to the amount of exact exchange (EXX), we employed functionals with different amount of EXX in order to establish the reliability of the results. We find that the SQ is somewhat more favored when decreasing the EXX to $10 \%$ (using the TPSSh functional $^{41}$ ), while increasing the EXX to 50\% (using BHLYP) or using the range-corrected CAM-B3LYP functional ${ }^{42}$ decreases the stability of SQ (Figure S12). This indicates that the B3LYP-D3/MM calculations might somewhat overestimate the stability of SQ. However, the overall conclusions remain the same with all functionals employed, validating the robustness of the results. We do not observe any linked deprotonation of the surrounding Tyr- 87 or His- 38 residues, but the eT process is coupled to a spontaneous reorganization to the hydrogenbonded conformation, further supporting the conformational reorganization of $\mathrm{Q}$ in the e $\mathrm{T}$ process. Interestingly, the e $\mathrm{T}$ also seems to couple to reorganization of the Arg-84 side chain near the N2 center that transiently forms a contact with the N6B center upon oxidation of the former (Figure S13).

In order to understand the role of Arg-84 and His-169 on N2 $\mathrm{pH}$ redox potentials, ${ }^{43-45}$ we prepared in silico H169M and R84A mutations and recomputed the $E_{\mathrm{m}}$ values at different $\mathrm{pHs}$ (Figure S14). We estimated in our models that R84A downshifts the $\mathrm{N} 2 E_{m}$ value by ca. $250 \mathrm{mV}$ and shows almost no $\mathrm{pH}$ dependence, while the H169 M mutation downshifts the $E_{\mathrm{m}}$ value by $150 \mathrm{mV}$ and has a $\mathrm{pH}$ dependence of ca. $30 \mathrm{mV} /$ $\mathrm{pH}$ unit. His-169 has previously been suggested to function as a redox Bohr group for N2, ${ }^{43,44}$ whereas our results suggest that a combined conformational change between Arg-84 and His-169 could also be linked to $\mathrm{N} 2$ reduction and in part contribute to the $\mathrm{pH}$ dependence of the $\mathrm{N} 2$ center. $^{45}$ The influence of other mutations ${ }^{43-45}$ could further shed light into the dynamics of the $\mathrm{eT}$ process and will be addressed in future work.

In order to address the dynamics of the second eT step from $\mathrm{N} 2$ to $\mathrm{SQ}$, we re-reduced the N2 center and initiated QM/MM MD simulations. We find that, upon deprotonation of His-169 $(\mathrm{N} \varepsilon)$, as suggested by our $\mathrm{PB}$ calculations (see above), the electron moves within 0.3 ps to the $S Q$ which in turn, further triggers a rapid deprotonation of His-38 and Tyr-87 on the ca. $0.3-1.3$ ps time scale, suggesting that the $\mathrm{pT}$ immediately follows the eT process (Figure 4). Interestingly, in the QM/

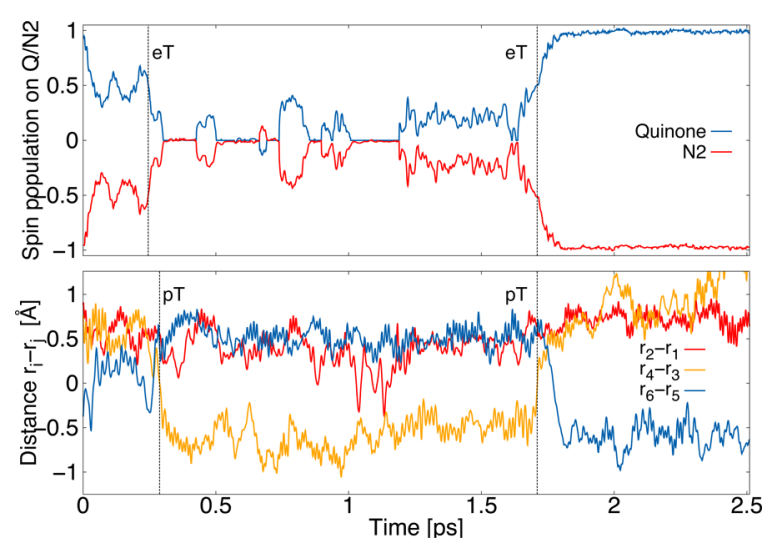

Figure 4. Dynamics of the second eT from N2 to SQ (top) upon deprotonation of His-169 and Tyr-87 (partial deprotonation at 1 ps, below). The eT process is coupled with $\mathrm{pT}$ from His-38 to SQ forming $\mathrm{QH}^{-}$.

$\mathrm{MM}$ trajectory, we also observe a back eT at around ca. $1.7 \mathrm{ps}$, which follows a similar but reverse PCET process, suggesting that the second eT from N2 to SQ is nearly isoenergetic; this further supports that the $E_{\mathrm{m}}$ of the $\mathrm{SQ} / \mathrm{QH}_{2}$ process is close to that of N2. We were not able to observe the second eT from N2 without deprotonation of His-169 (Figure S15), possibly due to the limited time scale of the simulations, whereas deprotonation of the $\mathrm{N} \delta$ proton on His-169 also resulted in a fast ( $>0.1 \mathrm{ps})$ eT process, coupled to a similar PCET from His38 and Tyr-87 (Figure S16). We could not identify likely proton donors that would participate in this putative deprotonation step, but we observe that the Q-binding site is accessible to water from the bulk (Figure S17). Our QM/MM $\mathrm{MD}$ simulations, which treat both the $\mathrm{eT}$ and $\mathrm{pT}$ processes between the $\mathrm{N} 2$ and $\mathrm{Q}$ quantum mechanically, thus support our previous results of the Q-site dynamics, suggesting that Tyr-87 and His-38 may act as local proton donors in the $\mathrm{QH}_{2}$ formation for the second eT step (Figure 5B). ${ }^{17}$ For the first eT step, our simulations suggest that SQ formation is not linked to proton uptake (Figure $5 \mathrm{~A}$ ). Our classical MD simulations of the resulting $\mathrm{QH}_{2} /$ deprotonated Tyr-87/His-38 state were further suggested to trigger conformational changes in the membrane domain $\mathrm{Nqo} 8$ that may in turn activate the proton pumping machinery. ${ }^{15}$

\section{CONCLUSIONS}

Despite significant structural and biochemical work on complex I, exact structural information on the Q-binding poses in its usual binding pocket, ca. $20 \AA$ above the membrane plane, has not been available. We have shown here by computational methods that both UQ and MQ can bind in two energetically possible binding modes to complex I: one with a hydrogenbonded interaction with Tyr-87/His-38 of subunit Nqo4, and 


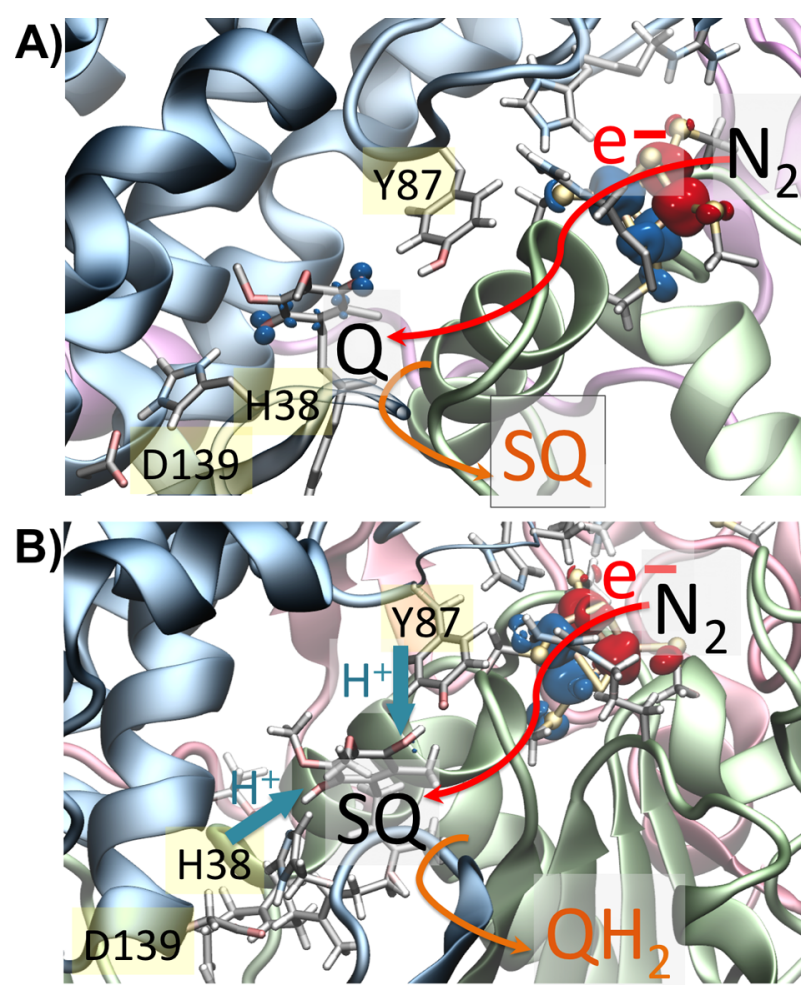

Figure 5. Sequence of eT (red arrow) and pT (blue arrow) between $\mathrm{N} 2$ and UQ based on QM/MM MD calculations. (A) First eT from $\mathrm{N} 2$ to UQ in the hydrogen-bonded conformation forming SQ. (B) Second electron moves from N2 to SQ and couples with $\mathrm{pT}$ from $\mathrm{H} 38$ and Y87, leading to formation of $\mathrm{QH}_{2} / \mathrm{QH}^{-}$.

one where the hydrogen bond with His-38 is replaced by a stacked conformation. The latter conformation was found to be energetically favored upon N2 reduction, and the Q-binding mode was further found to tune the $E_{\mathrm{m}}$ values as dispersive $\pi-\pi$ interactions cause smaller shifts in redox potentials relative to hydrogen bonds. We obtained a redox potential of $\mathrm{Q}$ that is unusually low due to its interaction with N2. Our QM/MM $\mathrm{MD}$ simulations of the first eT steps between the reduced $\mathrm{N} 2$ and $\mathrm{Q}$ suggest that $\mathrm{eT}$ is linked to a conformational change of $Q$ between stacked and hydrogen-bonded conformations that increases its electron affinity. The second eT step was further found to lead to a local PCET from Tyr-87/His-38 of subunit Nqo4 and is strongly favored by deprotonation of His-169 near the $\mathrm{N} 2$ center (Figure 4). Similar binding poses and $E_{\mathrm{m}}$ values were also observed for MQ. Our calculations suggest that redox tuning of $\mathrm{Q}$ could be important for controlling the rate of the terminal eT steps in complex I, while local PCET in the $Q$ chamber may in turn be responsible for triggering the proton pump in the membrane domain of complex I. Both eT steps between $\mathrm{N} 2$ and UQ were found to be nearly isoenergetic, suggesting that there might not be a large energy drop between $\mathrm{NADH}\left(E_{\mathrm{m}}=-320 \mathrm{mV}\right)$ and UQ/MQ $\left(E_{\mathrm{m}}<-300 \mathrm{mV}\right)$. Moreover, because both $\mathrm{Q}(+90 \mathrm{mV})$ and $\mathrm{MQ}(-80 \mathrm{mV})$ have higher $E_{\mathrm{m}}$ values in membranes, this further indicates that the main energy transduction event that is coupled to proton pumping could follow after $\mathrm{Q}$ release from its binding site near N2.

\section{COMPUTATIONAL METHODS}

DFT cluster and QM/MM models of complex I were built based on the X-ray structure of complex I from T. thermophilus (PDB ID:
4HEA). ${ }^{46}$ The model comprised subunits Nqo4, Nqo5, Nqo6, Nqo7, Nqo8, and Nqo9, the $\mathrm{Q}$ and three ISCs N2, N6B, and N6A (Figure S11). Protonation states based on the crystal structure were assigned by $\mathrm{PB}$ continuum electrostatics calculations. ${ }^{47,48}$ Classical MD simulations were performed by using the whole protein and considering the $\mathrm{UQ} / \mathrm{MQ}$ in oxidized (Q), SQ (i.e., $\mathrm{Q}^{\bullet-}$ ), and reduced $\left(\mathrm{Q}^{2-}\right)$ states, while the ISCs were fixed in their $\mathrm{N} 2[\mathrm{ox}]$ state $\left(2 \mathrm{Fe}^{3+} 2 \mathrm{Fe}^{2+}\right)$ with atomic partial charges that included the inorganic iron-sulfur and four ligated cysteine residues. For the $\mathrm{QM}$ calculations, we also considered the $\mathrm{N} 2$ cluster in its $\mathrm{N} 2$ [red] state $\left(1 \mathrm{Fe}^{3+} 3 \mathrm{Fe}^{2+}\right)$. The system was classically relaxed with NAMD2, ${ }^{49}$ for $10 \mathrm{~ns}$ at $T=310 \mathrm{~K}$ using a 1 fs integration time step with harmonic restrains on the $\mathrm{C} \alpha$ atoms and by employing the CHARMM27 force field. $^{50}$ The classical MD simulations were followed by QM/MM geometry optimization and first-principles dynamics for 5 ps for each state. The QM region consisted of three models: the N2 model, the $\mathrm{Q}$ model, and the combined N2-Q model. The N2 model comprised the N2 center and residues Cys-45, Cys-46, Cys-111, Cys-140, Arg-86, Arg-83, and His-169. The Q model comprised the $Q$ and residues His38, Asp-139, Thr-135, and Tyr-87, while the combined N2-Q model comprised both subsystems (see Figure S11). The QM/MM simulations were performed at the B3LYP-D3/def2-TZVP(Fe)/def2$\mathrm{SVP}(\mathrm{S}, \mathrm{C}, \mathrm{H}, \mathrm{N}, \mathrm{O})$ level, ${ }^{51-53}$ and the N2 center was modeled with antiferromagnetic coupling using the broken-symmetry spin-flip approach. ${ }^{33} \mathrm{QM}$ cluster models were optimized at the dispersion corrected B3LYP-D3/def2-TZVP(Fe, S)/def2-SVP (C, H, N, O). ${ }^{54,55}$ Single point energies were computed for all atoms at B3LYP-D3 and TPSSh-D3/def2-TZVPP levels (see Table S1). The QM models comprised the $\mathrm{Q}$ the N2 cluster, and residues His-38, Asp-139, Thr135, Tyr-87, Cys-45, Cys-46, Cys-111, Cys-140, Arg-86, and Arg-83 (Figure S4); terminal carbon atoms were fixed during the optimization process. Solvation effects were treated with $\operatorname{COSMO}^{56}$ with a dielectric constant $\varepsilon=4$. Calculations were performed with the CHARMM/TURBOMOLE ${ }^{57}$ interface, TURBOMOLE, ${ }^{58}$ and NWChem. ${ }^{59}$ VMD was used for visualization. ${ }^{60}$ Redox potentials were calculated based on electrostatic potentials obtained by solving numerically the linearized $\mathrm{PB}$ equation using $\mathrm{APBS}^{48,61}$ and $\mathrm{MC}$ titration sampling. ${ }^{47,62}$ The protein was described using atomic partial charges, embedded in an inhomogeneous dielectric continuum with dielectric constants of $\varepsilon_{\mathrm{p}}=4$ inside the protein and $\varepsilon_{\mathrm{w}}=80$ for the bulk water. The boundary interface between the protein and the solvent was calculated by the molecular surface routine implemented in APBS, using a solvent probe radius of $1.4 \AA$ and modeling an implicit ionic strength of $100 \mathrm{mM}$ potassium chloride. The redox potential was computed as a difference of electrostatic free energy shifts between a model compound in water and the model compound in the protein. ${ }^{25,62-64}$ To assess the influence of using a small or large model of the protein, we performed redox potential computations using the whole crystal structure and compared them with the small $\mathrm{QM} / \mathrm{MM}$ models observing only a small deviation of Q/SQ and N2 $E_{m}$ values of ca. $40 \mathrm{mV}$ (see Table S4). We also addressed the influence of the redox state of other ISCs on the Q/SQ and N2 values, suggesting that, in the current PB model, only N6B and N6A strongly influence the $\mathrm{Q} / \mathrm{SQ}$ and $\mathrm{N} 2 E_{m}$ values (Table S4). A reference value of the UQ (MQ) Q/SQ redox couple was estimated to $-188 \mathrm{mV}(-241$ $\mathrm{mV}$ ) in water by B3LYP/def2-TZVP calculation, based on the experimental $E_{\mathrm{m}}$ values of UQ and MQ in DMF and acetonitrile, ${ }^{25}$ (Table S5) by the approach of Knapp and co-workers. ${ }^{65-67}$ All our reported $E_{\mathrm{m}}$ values are given relative to the NHE.

\section{ASSOCIATED CONTENT}

\section{Supporting Information}

The Supporting Information is available free of charge on the ACS Publications website at DOI: $10.1021 /$ jacs.7b08486.

Detailed energetics, coordinates of $\mathrm{QM}$ and $\mathrm{QM} / \mathrm{MM}$ models, and supplementary figures and tables (PDF) Movie of the second eT (GIF) 


\section{AUTHOR INFORMATION}

\section{Corresponding Authors}

*ana.gamiz@tum.de

*ville.kaila@ch.tum.de

\section{ORCID}

Mikael P. Johansson: 0000-0002-9793-8235

Ville R. I. Kaila: 0000-0003-4464-6324

Notes

The authors declare no competing financial interest.

\section{ACKNOWLEDGMENTS}

We thank Prof. Mårten Wikström for insightful discussion on complex I and Dr. Adam Kubas for technical discussions. This project has received funding from the European Research Council (ERC) under the European Union's Horizon 2020 research and innovation program/grant agreement 715311. M.P.J. is supported by the Academy of Finland (289179). The Leibniz-Rechenzentrum (LRZ), SuperMuc (project: pr48de), and CSC-The Finnish IT Center for Science provided computational resources.

\section{REFERENCES}

(1) Brandt, U. Annu. Rev. Biochem. 2006, 75, 69-92.

(2) Sazanov, L. A.; Hinchliffe, P. Science 2006, 311, 1430-1436.

(3) Verkhovskaya, M.; Bloch, D. A. Int. J. Biochem. Cell Biol. 2013, 45, 491-511.

(4) Hirst, J. Annu. Rev. Biochem. 2013, 82, 551-575.

(5) Sazanov, L. A. Nat. Rev. Mol. Cell Biol. 2015, 16, 375-388.

(6) Wikström, M.; Sharma, V.; Kaila, V. R. I.; Hosler, J. P.; Hummer, G. Chem. Rev. 2015, 115, 2196-2221.

(7) Hirst, J.; Roessler, M. M. Biochim. Biophys. Acta, Bioenerg. 2016, 1857, 872-883.

(8) Verkhovskaya, M. L.; Belevich, N.; Euro, L.; Wikström, M.; Verkhovsky, M. I. Proc. Natl. Acad. Sci. U. S. A. 2008, 105, 3763-3767.

(9) Zickermann, V.; Kerscher, S.; Zwicker, K.; Tocilescu, M. A.; Radermacher, M.; Brandt, U. Biochim. Biophys. Acta, Bioenerg. 2009, 1787, 574-583.

(10) Hunte, C.; Zickermann, V.; Brandt, U. Science 2010, 329, 448451.

(11) Baradaran, R.; Berrisford, J. M.; Minhas, G. S.; Sazanov, L. A. Nature 2013, 494, 443-448.

(12) Zickermann, V.; Wirth, C.; Nasiri, H.; Siegmund, K.; Schwalbe, H.; Hunte, C.; Brandt, U. Science 2015, 347, 44-49.

(13) Fiedorczuk, K.; Letts, J. A.; Degliesposti, G.; Kaszuba, K.; Skehel, M.; Sazanov, L. A. Nature 2016, 538, 406-410.

(14) Zhu, J.; Vinothkumar, K. R.; Hirst, J. Nature 2016, 536, 354358.

(15) Wikström, M.; Hummer, G. Proc. Natl. Acad. Sci. U. S. A. 2012, 109, 4431-4436.

(16) Kaila, V. R. I.; Wikström, M.; Hummer, G. Proc. Natl. Acad. Sci. U. S. A. 2014, 111, 6988-6993.

(17) Sharma, V.; Belevich, G.; Gamiz-Hernandez, A. P.; Rog, T.; Vattulainen, I.; Verkhovskaya, M. L.; Wikström, M.; Hummer, G.; Kaila, V. R. I. Proc. Natl. Acad. Sci. U. S. A. 2015, 112, 11571-11576.

(18) Di Luca, A.; Gamiz-Hernandez, A. P.; Kaila, V. R. I. Proc. Natl. Acad. Sci. U. S. A. 2017, 114, E6314-E6321.

(19) de Vries, S.; Dörner, K.; Strampraad, M. J. F.; Friedrich, T. Angew. Chem., Int. Ed. 2015, 54, 2844-2848.

(20) Ohnishi, T. Biochim. Biophys. Acta, Bioenerg. 1998, 1364, 186.

(21) Tocilescu, M. A.; Fendel, U.; Zwicker, K.; Dröse, S.; Kerscher, S.; Brandt, U. Biochim. Biophys. Acta, Bioenerg. 2010, 1797, 625-632.

(22) Sinha, P. K.; Castro-Guerrero, N.; Patki, G.; Sato, M.; TorresBacete, J.; Sinha, S.; Miyoshi, H.; Matsuno-Yagi, A.; Yagi, T. Biochemistry 2015, 54, 753-764.

(23) Warren, J. J.; Tronic, T. A.; Mayer, J. M. Chem. Rev. 2010, 110, 6961-7001.
(24) Weinberg, D. R.; Gagliardi, C. J.; Hull, J. F.; Murphy, C. F.; Kent, C. A.; Westlake, B. C.; Paul, A.; Ess, D. H.; McCafferty, D. G.; Meyer, T. J. Chem. Rev. 2012, 112, 4016-4093.

(25) Prince, R. C.; Dutton, P. L.; Bruce, J. M. FEBS Lett. 1983, 160, 273-276.

(26) Gunner, M. R.; Madeo, J.; Zhu, Z. J. Bioenerg. Biomembr. 2008, 40, 509-519.

(27) Trumpower, B. Function of Quinones in Energy Conserving Systems; Academic Press: New York, 1982.

(28) Verkhovskaya, M.; Wikström, M. Biochim. Biophys. Acta, Bioenerg. 2014, 1837, 246-250.

(29) Ohnishi, T. Biochim. Biophys. Acta, Bioenerg. 1998, 1364, 186206.

(30) Yano, T.; Dunham, W. R.; Ohnishi, T. Biochemistry 2005, 44, 1744-1754.

(31) Ingledew, W. J.; Poole, R. K. Microbiol Rev. 1984, 48, 222-271.

(32) Noguchi, T.; Inoue, Y.; Tang, X. S. Biochemistry 1999, 38, 399403.

(33) Mouesca, J.-M.; Noodleman, L.; Case, D. A. Int. J. Quantum Chem. 1995, 56, 95-102.

(34) Brandt, U. Annu. Rev. Biochem. 2006, 75, 69-92.

(35) Verkhovsky, M.; Bloch, D. A.; Verkhovskaya, M. Biochim. Biophys. Acta, Bioenerg. 2012, 1817, 1550-1556.

(36) Zwicker, K.; Galkin, A.; Drose, S.; Grgic, L.; Kerscher, S.; Brandt, U. J. Biol. Chem. 2006, 281, 23013-7.

(37) Hanley, J.; Deligiannakis, Y.; MacMillan, F.; Bottin, H.; Rutherford, A. W. Biochemistry 1997, 36, 11543-11549.

(38) Cohen, A. J.; Mori-Sánchez, P.; Yang, W. Science 2008, 321, 792-794.

(39) Moser, C. C.; Keske, J. M.; Warncke, K.; Farid, R. S.; Dutton, P. L. Nature 1992, 355, 796-802.

(40) Page, C. C.; Moser, C. C.; Chen, X.; Dutton, P. L. Nature 1999, $402,47-52$

(41) Tao, J.; Perdew, J. P.; Staroverov, V. N.; Scuseria, G. E. Phys. Rev. Lett. 2003, 91, 146401.

(42) Yanai, T.; Tew, D. P.; Handy, N. C. Chem. Phys. Lett. 2004, 393, $51-57$.

(43) Zwicker, K.; Galkin, A.; Dröse, S.; Grgic, L.; Kerscher, S.; Brandt, U. J. Biol. Chem. 2006, 281, 23013-7.

(44) Grgic, L.; Zwicker, K.; Kashani-Poor, N.; Kerscher, S.; Brandt, U. J. Biol. Chem. 2004, 279, 21193.

(45) Kashani-Poor, N.; Zwicker, K.; Kerscher, S.; Brandt, U. J. Biol. Chem. 2001, 276, 24082-24087.

(46) Baradaran, R.; Berrisford, J. M.; Minhas, G. S.; Sazanov, L. A. Nature 2013, 494, 443-448.

(47) Kieseritzky, G.; Knapp, E. W. J. Comput. Chem. 2008, 29, 25752581.

(48) Baker, N. A.; Sept, D.; Joseph, S.; Holst, M. J.; McCammon, J. A. Proc. Natl. Acad. Sci. U. S. A. 2001, 98, 10037-10041.

(49) Phillips, J. C.; Braun, R.; Wang, W.; Gumbart, J.; Tajkhorshid, E.; Villa, E.; Chipot, C.; Skeel, R. D.; Kalé, L.; Schulten, K. J. Comput. Chem. 2005, 26, 1781-1802.

(50) MacKerell, A. D.; Bashford, D.; Bellott, M.; Dunbrack, R. L.; Evanseck, J. D.; Field, M. J.; Fischer, S.; Gao, J.; Guo, H.; Ha, S.; Joseph-McCarthy, D.; Kuchnir, L.; Kuczera, K.; Lau, F. T. K.; Mattos, C.; Michnick, S.; Ngo, T.; Nguyen, D. T.; Prodhom, B.; Reiher, W. E.; Roux, B.; Schlenkrich, M.; Smith, J. C.; Stote, R.; Straub, J.; Watanabe, M.; Wiórkiewicz-Kuczera, J.; Yin, D.; Karplus, M. J. Phys. Chem. B 1998, 102, 3586-3616.

(51) Becke, A. D. J. Chem. Phys. 1993, 98, 5648-5652.

(52) Lee, C. T.; Yang, W. T.; Parr, R. G. Phys. Rev. B: Condens. Matter Mater. Phys. 1988, 37, 785-789.

(53) Schäfer, A.; Horn, H.; Ahlrichs, R. J. Chem. Phys. 1992, 97, 2571-2577.

(54) Grimme, S.; Antony, J.; Ehrlich, S.; Krieg, H. J. Chem. Phys. 2010, 132, 154104

(55) Weigend, F.; Ahlrichs, R. Phys. Chem. Chem. Phys. 2005, 7, $3297-3305$ 
(56) Klamt, A.; Schüürmann, G. J. Chem. Soc., Perkin Trans. 2 1993, 5, 799-805.

(57) Riahi, S.; Rowley, C. N. J. Comput. Chem. 2014, 35, 2076-86.

(58) Ahlrichs, R.; Bär, M.; Häser, M.; Horn, H.; Kölmel, C. Chem. Phys. Lett. 1989, 162, 165-169.

(59) Valiev, M.; Bylaska, E. J.; Govind, N.; Kowalski, K.; Straatsma, T. P.; van Dam, H. J. J.; Wang, D.; Nieplocha, J.; Apra, E.; Windus, T. L.; de Jong, W. A. Comput. Phys. Commun. 2010, 181, 1477-1489.

(60) Humphrey, W.; Dalke, A.; Schulten, K. J. Mol. Graphics 1996, $14,33-38$.

(61) Holst, M.; Saied, F. J. Comput. Chem. 1993, 14, 105-113.

(62) Rabenstein, B.; Ullmann, G. M.; Knapp, E. W. Eur. Biophys. J. 1998, 27, 626-637.

(63) Ishikita, H.; Knapp, E. W. J. Am. Chem. Soc. 2004, 126, 80598064.

(64) Zhu, Z.; Gunner, M. R. Biochemistry 2005, 44, 82-96.

(65) Schmidt am Busch, M.; Knapp, E. W. J. Am. Chem. Soc. 2005, $127,15730-15737$.

(66) Galstyan, A. S.; Knapp, E. W. J. Comput. Chem. 2009, 30, 203211.

(67) Gamiz-Hernandez, A. P.; Galstyan, A. S.; Knapp, E. W. J. Chem. Theory Comput. 2009, 5, 2898-2908. 